\title{
Influence of resonance decays on triangular flow in heavy-ion collisions
}

\author{
J. Crkovská ${ }^{1,2, a}$, L. V. Bravina ${ }^{2}$, G. Kh. Eyyubova ${ }^{1,3}$, and E. E. Zabrodin ${ }^{2,3}$ \\ ${ }^{1}$ Faculty of Nuclear Sciences and Physical Engineering, Czech Technical University in Prague, Břehová 7 , \\ 11000 Prague, Czech Republic \\ ${ }^{2}$ Department of Physics, University of Oslo, P. O. Box 1048 Blindern, N-0316, Oslo, Norway \\ ${ }^{3}$ Skobeltsyn Institute of Nuclear Physics, Lomonosov Moscow State University, Leninskie Gory, GSP-1, \\ Moscow 119991, Russian Federation
}

\begin{abstract}
Anisotropic flow in relativistic collisions of heavy-ions yields important information about the state of the hot and dense matter created in the collision. Study of triangular flow in $\mathrm{Pb}+\mathrm{Pb}$ collisions at $\mathrm{LHC}$ was performed using HYDJET++ Monte Carlo generator. HYDJET++ combines both hydrodynamics-driven soft part together with hard jet-part, giving a realistic prediction for vast number of hadron species. The model also enables study of influence of final-state interactions on flow of created hadrons. Triangular flow patterns of pions, kaons and protons were studied. We found that resonance decays influence significantly the shape of the distributions.
\end{abstract}

\section{Introduction}

Relativistic heavy-ion collisions present a unique tool for study of matter under extreme conditions. The high temperatures and energy densities give rise to new state of matter known as the quark-gluon plasma (QGP). The thermalised hot and dense matter does expand and cool down until it reaches critical temperature and goes through a phase transition into a hadronic matter. The freeze-out does occur very shortly after the initial collisions, in order of $\sim 10 \mathrm{ps.} \mathrm{Hence} \mathrm{the} \mathrm{QGP} \mathrm{cannot} \mathrm{be}$ detected directly, one would need some signatures that would permit further studies of this new exotic phase of matter.

Flow is a collective motion of matter created in collision, created by the pressure gradient inside the fireball, thus giving additional boost to the out-coming particles. The azimuthal distribution of created particles emitted at angle $\phi$ with respect to reaction plane $\Psi_{R}$ can be expanded into a Fourier series as

$$
\frac{\mathrm{d} N}{\mathrm{~d} \varphi}=\frac{N}{2 \pi}\left[1+2 \sum v_{n} \cos \left(n\left(\varphi-\Psi_{R}\right)\right)\right]
$$

where the Fourier coefficients are in a form

$$
v_{n}=\left\langle\cos \left[n\left(\phi-\Psi_{R}\right)\right]\right\rangle .
$$

a. e-mail: crkovjan@fjfi.cvut.cz 
The elliptic flow $v_{2}$ gives the dominant contribution to anisotropic flow in peripheral and semiperipheral collisions. $v_{2}$ arises from the asymmetry of the overlap zone. As the spatially anisotropic fireball evolves, it resumes spatial symmetry, thus giving rise to momentum anisotropy of out-coming particles. Hence it is a self-quenching phenomenon which takes place early after the collision when the QGP is still present. However the actual overlap zone fluctuates event-by-event around the geometrical zone. Triangular flow $v_{3}$ originates from such initial fluctuations.

Number-of-constituent-quarks (NCQ) scaling is a prominent feature of elliptic flow, first observed in 2007 at RHIC [2]. Elliptic flow of identified hadron species was divided by number of quarks inside of each species and ploted as a function of transverse kinetic energy. The resulting distributions were of identical shape for all studied species. Recent results from STAR suggest that $v_{3}$ scales as $v_{3} / n_{q}^{3 / 2}\left(k E_{T} / n_{q}\right)[3]$.

\section{HYDJET++}

HYDJET++ [4] stands for HYDrodynamics with JETs and is a Monte Carlo heavy-ion event generator. It is a superposition of hydrodynamical part and jet part. Each part is treated independently. Among the advantages of this model is its efficiency, its ability to reproduce realistic shapes of distributions and that it includes a vast table of resonances.

In the soft part, the hadrons are generated on freeze-out hyper-surface described by a parametrisation of relativistic hydrodynamics with given initial conditions and equation of state. The jets are generated from binary collisions at given impact parameter $b$ from Glauber model. Only partons fulfilling the condition $p_{T}>p_{T}^{\min }$ are further evolved. Those with transverse momentum lower than the threshold value are thermalised and their hadronisation products are included in the soft component.

The elliptic flow is generated through spatial anisotropy $\varepsilon(b)$ and momentum anisotropy $\delta(b)$ as follows:

$$
\begin{aligned}
\varepsilon(b) & =\frac{R_{y}^{2}-R_{x}^{2}}{R_{y}^{2}+R_{x}^{2}} \\
\frac{u^{y}}{u^{x}} & =\sqrt{\frac{1-\delta(b)}{1+\delta(b)}} \tan \phi
\end{aligned}
$$

where $R_{x}$ and $R_{y}$ are the projection of the fireball radius onto the x-direction and y-direction respectively and $u^{x, y}$ is the fluid velocity in corresponding direction. The anisotropy parameters $\varepsilon(b)$ and $\delta(b)$ were obtained by fitting the experimental data.

To obtain triangular flow, the radius of the freeze-out surface has to be modified using an additional triangularity parameter $\varepsilon_{3}(b)$ as

$$
R(b, \phi)=R_{f}(b) \frac{\sqrt{1-\varepsilon^{2}(b)}}{\sqrt{1-\varepsilon(b) \cos 2 \phi}}\left\{1+\varepsilon_{3}(b) \cos \left[3\left(\phi-\Psi_{3}\right)\right]\right\}
$$

The axis $\Psi_{3}$ is generated randomly w.r.t. $\Psi_{2}$, therefore, elliptic and triangular flows are uncorrelated.

\section{Triangular flow at LHC}

We performed a study of the influence of decays of the resonances on triangular flow at LHC energies. HYDJET++ was tuned to give realistic shapes of $v_{2}$ and $v_{3}$ distributions [5]. In Fig. 1 the generated $v_{3}$ distribution for charged particles (protons, pions and kaons) in $\mathrm{Pb}+\mathrm{Pb}$ collisions at 


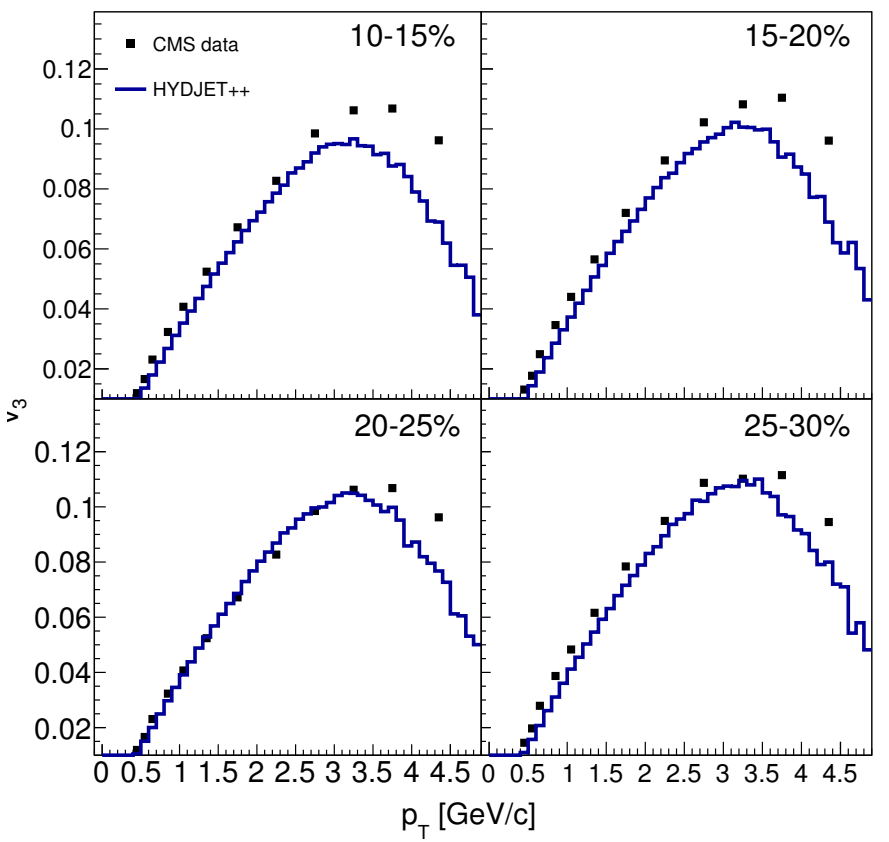

Figure 1. Triangular flow of charged particles in $\mathrm{Pb}+\mathrm{Pb}$ at $\sqrt{s_{N N}}=2.76 \mathrm{TeV}$. Histograms are HYDJET++ calculations, squares are CMS data [6].

$\sqrt{s_{N N}}=2.76 \mathrm{TeV}$ is compared with CMS data [6]. The model provides a particularly good description of data in softer region. The overall shape of the distribution corresponds to the trend observed in experiment.

Transverse momentum distributions of triangular flow $v_{3}\left(p_{T}\right)$ are shown in Fig. 2. Black dots show triangular flow distribution for all charged particles calculated with HYDJET ++ . Blue solid line shows flow obtained from the hydro-part of the model. The observed rise in flow is due to hydrodynamics, however already at low $p_{T}$, the real distribution starts to deviate from the hydro-calculation. At high $p_{T}$ the majority of particles are produced from jets, which carry little flow (see black dashed line), thus explaining the fall in the distribution. The distribution for direct charged particles (red dashed line) follows a pattern similar to all charged particles.

Upper plot in Fig 3 shows calculation of $v_{3}\left(p_{T}\right)$ for protons, pions and kaons. The patterns show a meson-baryon branching in soft region and branch-crossing at higher $p_{T}$ which is indeed observed experimentally. Mass ordering is also apparent, as the lightest particles resist to the push from medium the least. Bottom plot pictures $v_{3}\left(p_{T}\right)$ for protons, pions and kaons coming from hydrodynamics within HYDJET++. Mass-ordering and branching can be explained by hydrodynamics, however to fully describe the shape of the total distribution and the branch-crossing, another mechanism needs to be taken into account.

Fig. 4 shows $v_{3}\left(p_{T}\right)$ for charged particles in $\mathrm{Pb}+\mathrm{Pb}$ at $\sqrt{s_{N N}}=2.76 \mathrm{TeV}$, calculated with $\mathrm{HY}$ DJET++. Calculations were carried in 4 centrality bins within the range of $0-40 \%$. Full circles represent flow of all charged particles while open circles show flow of direct charged particles. The effect of resonance decays is significant both in magnitude and in position of the maximum of distributions. The maximum of the distribution is shifted to higher $p_{T}$ of about $10 \%$ while the difference of amplitude is about $40 \%$ in all centrality bins. 


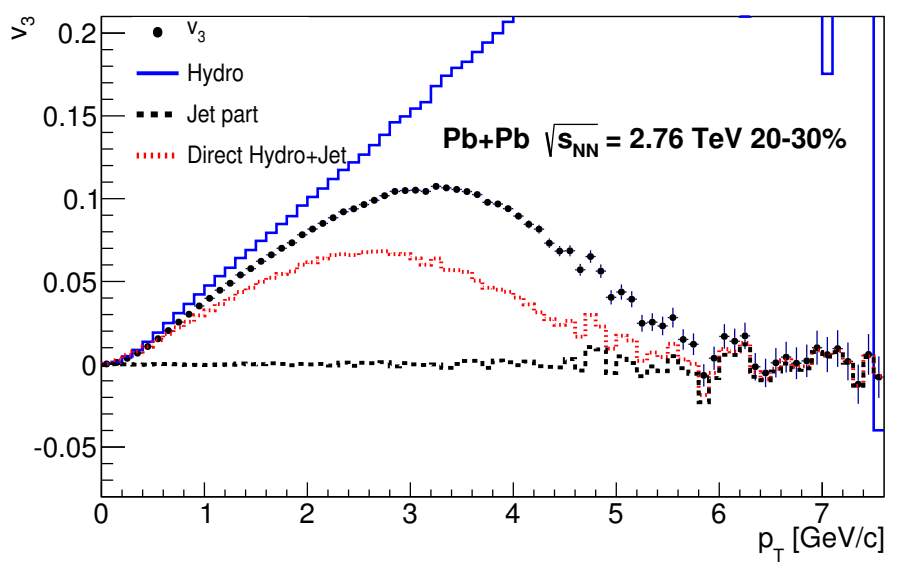

Figure 2. Transverse momentum distributions for $v_{3}$ calculated with HYDJET++ for charged particles in $\mathrm{Pb}+\mathrm{Pb}$ at $\sqrt{s_{N N}}=2.76 \mathrm{TeV}$. Black circles show $v_{3}\left(p_{T}\right)$ for all charged particles, histograms represent flow coming from the hydrodynamics (blue solid line) and from jets (black dashed line) respectively. Red dotted histogram shows $v_{3}\left(p_{T}\right)$ for direct charged particles.

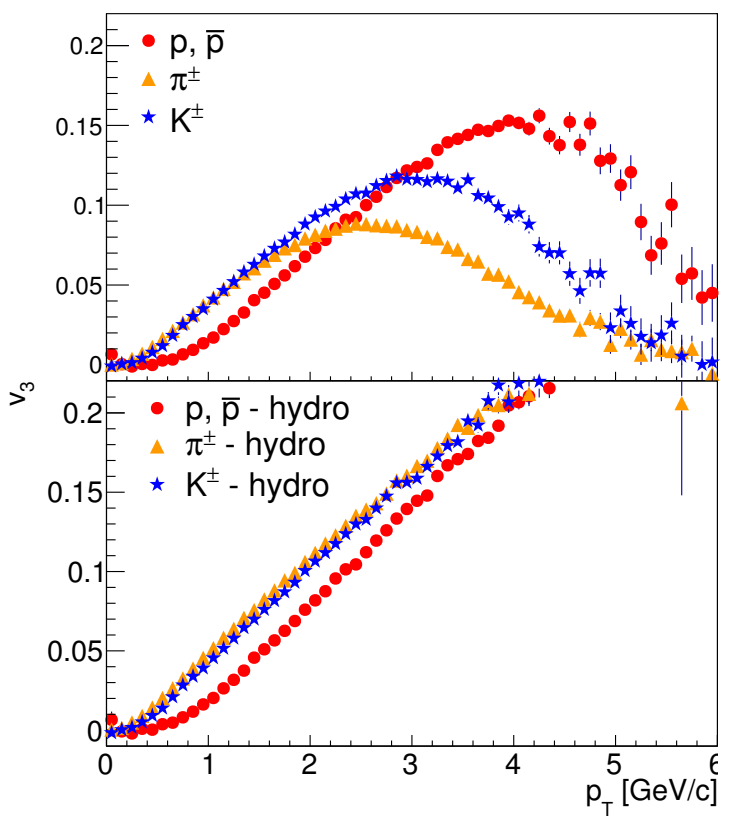

Figure 3. Top: Transverse momentum distribution of triangular flow $v_{3}\left(p_{T}\right)$ for all pions (yellow triangles), protons (red circles) and kaons (blue stars) in $\mathrm{Pb}+\mathrm{Pb}$ at $\sqrt{s_{N N}}=2.76 \mathrm{TeV}$ calculated with HYDJET++. Bottom: $v_{3}\left(p_{T}\right)$ distributions for pions, protons and kaons obtained from the hydrodynamical part within HYDJET++.

NCQ scaling of triangular flow at LHC was also studied. We considered the $v_{3}$ scaling as $v_{3} / n_{q}^{3 / 2}$, which was recently suggested by STAR [3]. Results for protons, kaons and pions are shown in Fig. $5(\mathrm{a}, \mathrm{b})$ for all hadrons and direct hadrons respectively. Plots $(\mathrm{c}, \mathrm{d})$ show the distributions $(\mathrm{a}, \mathrm{b})$ respectively divided by the corresponding distribution for proton. Proton and pion distributions seem to follow the scaling at low $p_{T}$, however the kaon distribution deviates by more than $50 \%$. In case of direct hadrons, there is no evident scaling pattern as the pions do also deviate significantly already in the soft region. 

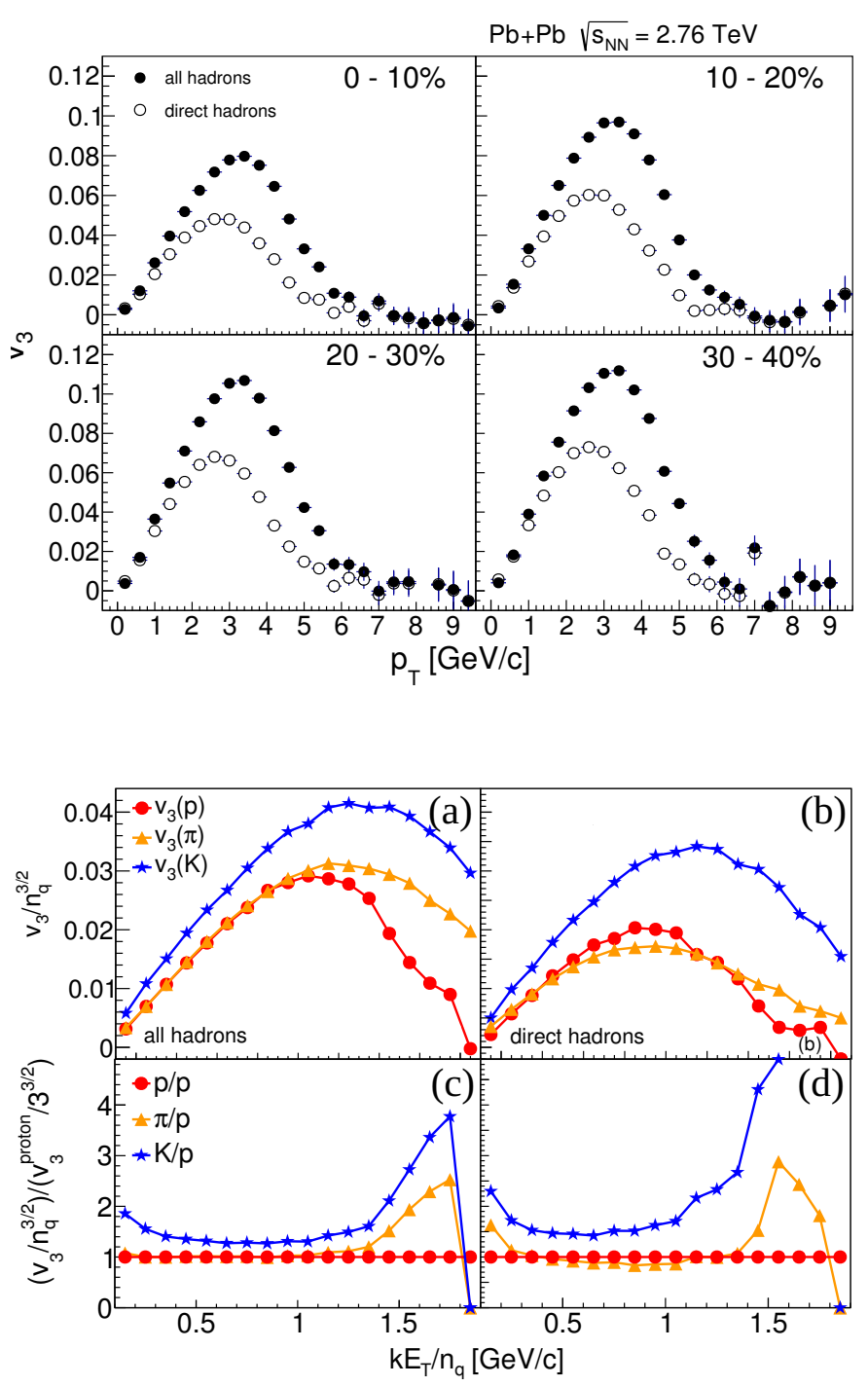

Figure 4. Triangular flow distribution $v_{3}\left(p_{T}\right)$ for all charged particles (full circles) and direct charged particles (open circles) in $\mathrm{Pb}+\mathrm{Pb}$ at $\sqrt{s_{N N}}=2.76 \mathrm{TeV}$ at centrality $0-40 \%$.

\section{Figure 5.}

Number-of-constituent-quarks scaling for triangular flow in $\mathrm{Pb}+\mathrm{Pb}$ at $\sqrt{s_{N N}}=2.76 \mathrm{TeV}$. Distributions for all protons (red circles), pions (yellow triangles) and kaons (blue stars) are shown in (a). The same for direct particles is in (b). The distributions of protons, pions and kaons divided by the proton distribution are depicted in (c), (d) shows the same for direct particles.

\section{Conclusions}

We performed a study of the effect of resonance decays on the triangular flow in $\mathrm{Pb}+\mathrm{Pb}$ collisions within the HYDJET++ framework. The model provides a fair description of $v_{3}$ with centrality. It was found that resonances influence significantly the shape of flow distributions: for $v_{3}$ we observed in maximum a shift of about $10 \%$ to higher $p_{T}$ and growth of about $40 \%$ in amplitude.

The number-of-constituent quark scaling is broken at LHC for $v_{3}$ as well as for $v_{2}$. However, the resonance decays drive the flow towards scaling.

It is vital to further study the flow of mother and daughter particles as well as influence of jets to better understand the dynamics of the system. 


\section{Acknowledgement}

This work was supported by the grant of the Grant Agency of Czech Republic n.13-20841S and by the Grant Agency of the Czech Technical University in Prague, grant No. SGS13/215/OHK4/3T/14.

\section{References}

[1] K. Aamodt et al. (ALICE Collaboration), Phys. Rev. Lett 107 (2011) 032301.

[2] A. Adare et al. (PHENIX Collaboration), Phys. Rev. Lett 98 (2007) 162301.

[3] X. Sun (for STAR Collaboration), J. Phys.: Conf. Ser. 535 (2014) 012005.

[4] I. P. Lokhtin et al., Comp. Phys. Comm. 180 (2009) 779-799.

[5] L. V. Bravina et al., Eur. Phys. J. C 74 (2014) 2807.

[6] S. Chatrchyan et al. (CMS Collaboration), Phys. Rev. C 87 (2013) 014902. 